\title{
El cambio del perfil epidemiológico de la mortalidad materna en Chile dificultará el cumplimiento del $5^{\circ}$ objetivo del Milenio
}

\author{
ENRIQUE DONOSO S., JORGE A. CARVAJAL C.
}

\section{The change in the epidemiological profile of maternal mortality in Chile will hinder the fulfillment of the Millennium $5^{\text {th }}$ goal}

Background: The death of women during pregnancy, childbirth or puerperium, remains a serious public health problem worldwide. Chile is committed to comply with the Millennium $5^{\text {th }}$ Goal of reducing maternal mortality to 9.9/100,000 live births in 2015. Aim: To analyze trends in maternal mortality in Chile during 2000-2009. Material and Methods: A descriptive population analysis using raw data obtained from the yearbooks of the National Institute of Statistics of Chile. Maternal mortality, causes of death and age of the dead mothers were evaluated. The causes of maternal death were classified according to the tenth revision of International Classification of Diseases. Trend studies were performed using Pearson correlation analysis. Results: In the studied period there were no significant changes in maternal mortality and fertility. The five major causes of maternal death were concurrent diseases, hypertension, abortion, obstetric embolism and postpartum hemorrhage. Mortality associated with concurrent illness showed a significant upward trend $(r=0.656, p=0.035)$. Abortion associated mortality had a significant downward trend $(r=-0.712, p=0.023)$. The group of women 40 years and older significantly increased its birth rate $(r=0.930$, $p<0.001$ ), this group showed the highest maternal mortality, especially in association with concurrent diseases. Conclusions: The increased birth rate occurring in women over 40 years old and its larger maternal mortality rate, probably will hinder the fulfillment of the Millennium $5^{\text {th }}$ goal in Chile.

(Rev Med Chile 2012; 140: 1253-1262).

Key words: Chile; Maternal mortality; Maternal welfare.

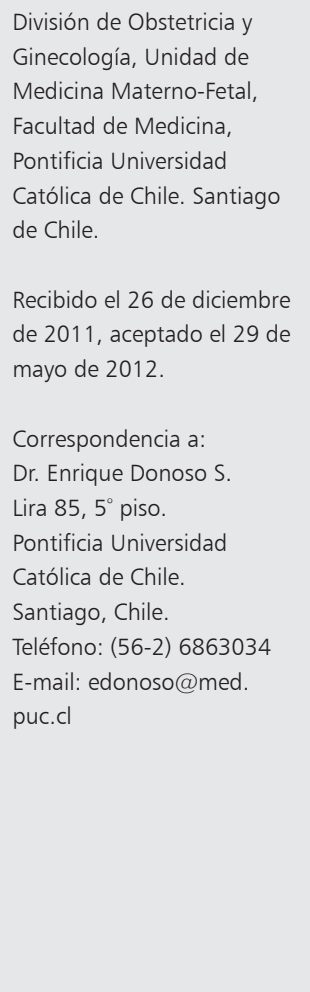

L a muerte de la mujer durante el embarazo, parto o puerperio, sigue siendo un grave problema mundial de salud pública, especialmente en los países en desarrollo ${ }^{1,2}$. Desde mediados del siglo XX, se han efectuado compromisos a nivel regional y mundial ${ }^{3}$, destinados a reducir las muertes maternas, entre otros objetivos sanitarios y sociales. El último de estos compromisos, es liderado por las Naciones Unidas, denominado " $\mathrm{Ob}$ jetivos de Desarrollo del Milenio"4, que establece en su $5^{\circ}$ objetivo reducir la mortalidad materna en $75 \%$ para el período $1990-2015$.
En 1960, Chile, presentaba una mortalidad materna de 299/100.000 nacidos vivos, una de las más altas de la Región de las Américas 5 . En esa época, la primera causa de muerte materna eran las complicaciones del aborto, alcanzando a un tercio del total de las muertes ${ }^{6}$. A partir de esa década, la introducción de los métodos anticonceptivos permitió a la mujer chilena un mejor control de su fecundidad, llevando a la reducción de las muertes maternas por aborto ${ }^{5,7}$. El continuo desarrollo social, económico y sanitario del país, permitió reducir la mortalidad materna en más de $50 \%$ en 
el período 1990-2000, especialmente por la reducción significativa de las muertes maternas atribuibles a aborto, hipertensión arterial y sepsis puerperal ${ }^{8}$, cumpliendo con el Plan de Acción Regional para la Reducción de la Mortalidad Materna en las Américas?.

Ese logro hizo muy promisorio el cumplimiento del $5^{\circ}$ objetivo del Milenio, de ahí que el Gobierno de Chile estableció dentro de los objetivos sanitarios para la década (2000-2010) una mortalidad materna de $12 / 100.000$ nacidos vivos al $2010^{10} \mathrm{y}$ de $9,9 / 100.000$ nacidos vivos al $2015^{4}$.

El objetivo de este estudio es analizar la tendencia de la mortalidad materna en Chile en el período 2000-2009 y los factores epidemiológicos que pudieran estar involucrados en el cumplimiento del $5^{\circ}$ objetivo del Milenio.

\section{Materiales y Método}

Se diseñó un estudio observacional y descriptivo para analizar la mortalidad materna en Chile entre los años 2000 y 2009. Las variables evaluadas fueron la razón de mortalidad materna (RMM), las causas de muerte, la edad de las madres fallecidas y la natalidad expresada en el número de nacidos vivos (nv). Los datos brutos se obtuvieron de los anuarios de estadísticas vitales publicados por el Instituto Nacional de Estadísticas de Chile ${ }^{11}$.

Se definió muerte materna al fallecimiento de la mujer durante el embarazo, parto o puerperio, por causas propias de la gestación o agravadas por esta, excluidas las causas accidentales e incidentales. La RMM se expresó como el número de muertes maternas por 100.000 nv. La RMM por año de ocurrencia y causa de muerte, se calculó en relación al número de nacidos vivos corregidos (nacidos vivos inscritos en el año de nacimiento, más los inscritos en los primeros tres meses del año siguiente). La natalidad y RMM según edad materna se analizó en relación a los nacidos vivos inscritos en el año de nacimiento. Para su análisis, las causas de muerte materna se clasificaron según la décima revisión de la Clasificación Estadística Internacional de
Enfermedades y Problemas Relacionados con la Salud (CIE 10), contenidas entre los códigos O00-O99912.

Para el análisis por causas de muerte, el código O99 fue definido como enfermedades concurrentes, que corresponde a todas las afecciones que son agravadas por el embarazo, excepto las que afectan directamente al feto, las enfermedades infecciosas o parasitarias, las causas externas y las que tienen codificación específica ${ }^{12}$. La tendencia y las fluctuaciones de las variables se estudiaron respectivamente por medio del análisis de correlación de Pearson (2 colas) y el porcentaje de cambio acumulado. Se estableció como nivel de significación estadística un valor $\mathrm{p}<0,05$.

\section{Resultados}

En el período 2000-2009, se registró un total de 2.419.834 nacidos vivos corregidos, 431 muertes maternas y una RMM promedio de 17,8/100.000 nv (Tabla 1 ). No hubo cambios significativos en la tendencia de la RMM ( $\mathrm{r}=-0,030 ; \mathrm{p}=0,934)$, de las muertes maternas $(\mathrm{r}=-0,052 ; \mathrm{p}=0,888)$ y del número de nacimientos $(\mathrm{r}=+0,013 ; \mathrm{p}=0,971)$, con un aumento acumulado de la RMM de 0,23\%, de las muertes maternas de 0,49\% y de la natalidad de $0,79 \%$.

La distribución por rangos de edad materna de la natalidad mostró que $15,9 \%$ correspondió a menores de 20 años, el $68,2 \%$ a mujeres entre $20-34$ años y $15,9 \%$ a mujeres de 35 o más años (Tabla 2). No hubo cambios

\section{Tabla 1. Número de muertes maternas, nacidos vivos corregidos y razón de mortalidad materna, Chile 2000-2009}

\begin{tabular}{|lccc|}
\hline Año & $\begin{array}{c}\text { Muertes } \\
\text { maternas } \\
\text { (n) }\end{array}$ & $\begin{array}{c}\text { Nacidos vivos } \\
\text { corregidos } \\
\text { (n) }\end{array}$ & $\begin{array}{c}\text { Mortalidad } \\
\text { materna } \\
\text { (x 100.000 nv) }\end{array}$ \\
\hline 2000 & 49 & 252.155 & 19,43 \\
2001 & 45 & 248.651 & 18,10 \\
2002 & 42 & 241.027 & 17,43 \\
\hline 2003 & 30 & 236.223 & 12,70 \\
\hline 2004 & 42 & 232.588 & 18,06 \\
\hline 2005 & 48 & 232.092 & 20,68 \\
\hline 2006 & 47 & 233.104 & 20,16 \\
\hline 2007 & 44 & 242.054 & 18,18 \\
\hline 2008 & 41 & 248.366 & 16,51 \\
\hline 2009 & 43 & 253.574 & 16,96 \\
\hline $2000-2009$ & 431 & 2.419 .834 & 17,81 \\
\hline
\end{tabular}


significativos en la tendencia de la natalidad en todos los rangos de edad, con excepción de las mujeres de 40 o más años que presentaron una tendencia ascendente y significativa $(r=0,930$; $\mathrm{p}<0,001)$.

Tabla 2. Análisis de la tendencia de la natalidad, Chile 2000-2009

\begin{tabular}{|c|c|c|c|c|}
\hline $\begin{array}{c}\text { Edad } \\
\text { (años) }\end{array}$ & $\begin{array}{c}\text { Nacidos } \\
\text { n }\end{array}$ & $\begin{array}{l}\text { rivos } \\
(\%)\end{array}$ & $r=$ & Valor $\mathbf{p}$ \\
\hline$<15$ & 10.176 & $(0,4)$ & $-0,372$ & $0,290 *$ \\
\hline 15-19 & 371.967 & $(15,5)$ & 0,252 & $0,482^{*}$ \\
\hline $20-24$ & 559.289 & $(23,3)$ & 0,410 & $0,239 *$ \\
\hline $25-29$ & 576.205 & $(24,0)$ & $-0,370$ & 0,292 * \\
\hline $30-34$ & 501.767 & $(20,9)$ & $-0,378$ & 0,281 * \\
\hline $35-39$ & 296.461 & $(12,4)$ & 0,347 & $0,327^{*}$ \\
\hline$\geq 40$ & 84.345 & $(3,5)$ & 0,930 & 0,001 \\
\hline
\end{tabular}

*No significativo. r: coeficiente de correlación de Pearson (2 colas).
Las causas de muerte materna con más de 15 casos fueron: 115 muertes $(26,7 \%)$ por enfermedades maternas concurrentes (código O99); 105 muertes $(24,4 \%)$ por hipertensión arterial (códigos O10-O16); 38 muertes $(8,8 \%)$ por aborto (códigos O03-O06); 21 muertes (4,9\%) por embolia obstétrica (código O88), 20 muertes (4,6\%) por hemorragia posparto (código O72), 20 muertes $(4,6 \%)$ por embarazo ectópico (código O00) y 16 muertes $(3,7 \%)$ por sepsis puerperal (código O85) (Tabla 3). Las primeras dos causas fueron responsables de $51,1 \%$ del total de muertes maternas.

La RMM para las primeras 7 causas de muerte materna fueron: enfermedades concurrentes 4,8/100.000 nv, hipertensión arterial 4,3/100.000 nv, aborto $1,6 / 100.000 \mathrm{nv}$, embolia obstétrica $0,9 / 100.000 \mathrm{nv}$, hemorragia posparto $0,8 / 100.000$ nv, embarazo ectópico $0,8 / 100.000$ nv y sepsis puerperal 0,7/100.000 nv.

La tendencia de la RMM por enfermedades concurrentes $(r=0,656 ; p=0,035)$ fue ascen-

Tabla 3. Muertes maternas según causas (CIE 10), Chile 2000-2009

\begin{tabular}{|c|c|c|}
\hline Código & Causas & $\mathbf{n}$ \\
\hline $000-008$ & Embarazo terminado en aborto & 59 \\
\hline O०0 & Embarazo ectópico & 20 \\
\hline 001 & Mola hidatidiforme & 1 \\
\hline 002 & Otros productos anormales de la concepción & 2 \\
\hline O03 & Aborto espontáneo & 4 \\
\hline 004 & Aborto médico & 1 \\
\hline 005 & Otro aborto & 1 \\
\hline 006 & Aborto no especificado & 29 \\
\hline 008 & Complicaciones consecutivas al aborto, al embarazo ectópico y al embarazo molar & 1 \\
\hline 010-016 & Edema, proteinuria y trastornos hipertensivos en el embarazo, el parto y el puerperio & 105 \\
\hline 010 & Hipertensión preexistente que complica el embarazo, parto y el puerperio & 3 \\
\hline 011 & Trastornos hipertensivos preexistentes, con proteinuria agregada & 1 \\
\hline 013 & Hipertensión gestacional (inducida por el embarazo) sin proteinuria significativa & 11 \\
\hline 014 & Hipertensión gestacional (inducida por el embarazo) con proteinuria significativa & 38 \\
\hline 015 & Eclampsia & 49 \\
\hline 016 & Hipertensión materna no especificada & 3 \\
\hline $020-029$ & Otros trastornos maternos relacionados principalmente con el embarazo & 21 \\
\hline $\mathrm{O} 21$ & Vómitos excesivos en el embarazo & 1 \\
\hline $\mathrm{O} 22$ & Complicaciones venosas en el embarazo & 3 \\
\hline $\mathrm{O} 23$ & Infección de las vías genitourinarias en el embarazo & 2 \\
\hline $\mathrm{O} 24$ & Diabetes mellitus & 6 \\
\hline $\mathrm{O} 26$ & Atención a la madre por otras complicaciones principalmente relacionadas con el embarazo & 9 \\
\hline
\end{tabular}


Tabla 3. Muertes maternas según causas (CIE 10), Chile 2000-2009 (continuación)

\begin{tabular}{|c|c|c|}
\hline Código & Causas & $\mathbf{n}$ \\
\hline $030-048$ & $\begin{array}{l}\text { Atención materna relacionada con el feto y la cavidad amniótica y con posibles } \\
\text { problemas del parto }\end{array}$ & 14 \\
\hline 041 & Otros trastornos del líquido amniótico y de las membranas & 7 \\
\hline O44 & Placenta previa & 6 \\
\hline 045 & Desprendimiento prematuro de la placenta (abruptio placentae) & 1 \\
\hline $060-075$ & Complicaciones del trabajo de parto y del parto & 49 \\
\hline 062 & Anormalidades de la dinámica del trabajo de parto & 10 \\
\hline 066 & Otras obstrucciones del trabajo de parto & 1 \\
\hline O67 & Trabajo de parto y parto complicados por hemorragia intraparto, no clasificados en otra parte & 1 \\
\hline 071 & Otro trauma obstétrico & 5 \\
\hline 072 & Hemorragia posparto & 20 \\
\hline 075 & Otras complicaciones del trabajo de parto y del parto, no clasificadas en otra parte & 12 \\
\hline $080-084$ & Parto & 2 \\
\hline O82 & Parto único por cesárea & 2 \\
\hline 085-092 & Complicaciones principalmente relacionadas con el puerperio & 48 \\
\hline 085 & Sepsis puerperal & 16 \\
\hline O86 & Otras infecciones puerperales & 2 \\
\hline O87 & Complicaciones venosas en el puerperio & 3 \\
\hline O88 & Embolia obstétrica & 21 \\
\hline 089 & Complicaciones de la anestesia durante el puerperio & 2 \\
\hline 090 & Complicaciones del puerperio, no clasificadas en otra parte & 4 \\
\hline 094-099 & Otras afecciones obstétricas no clasificadas en otra parte & 133 \\
\hline 095 & Muerte obstétrica de causa no especificada & 5 \\
\hline 096 & $\begin{array}{l}\text { Muerte materna debida a cualquier causa obstétrica que ocurre después de los } 42 \text { días pero antes de } \\
1 \text { año del parto }\end{array}$ & 3 \\
\hline 098 & $\begin{array}{l}\text { Enfermedades maternas infecciosas y parasitarias clasificables en otra parte pero que complican el } \\
\text { embarazo, el parto y el puerperio }\end{array}$ & 10 \\
\hline 099 & $\begin{array}{l}\text { Otras enfermedades maternas clasificables en otra parte, pero que complican el embarazo, parto y } \\
\text { el puerperio }\end{array}$ & 115 \\
\hline 000-099 & Total & 431 \\
\hline
\end{tabular}

dente y significativa, mientras que la tendencia de la RMM por aborto $(r=-0,712 ; p=0,023)$ fue significativamente descendente. No hubo cambios significativos en la tendencia de la RMM por hipertensión arterial $(\mathrm{r}=-0,186 ; \mathrm{p}=0,273)$ y embolia obstétrica $(r=0,182 ; p=0,351)$. No se efectuó análisis de tendencia para hemorragia posparto, embarazo ectópico y sepsis puerperal por presentar 20 o menos casos de muertes en el período estudiado.

La RMM por rangos de edad mostró una tendencia ascendente a mayor edad materna, con punto de quiebre desde los 35 años (Figura 1). La mayor RMM correspondió a las mujeres de
45-49 años (104,9/100.000 nv) y la menor a las adolescentes menores de 15 años (9,8/100.000 nv). La mortalidad materna en mujeres menores de 35 años fue inferior a 20/100.000 nv. Del total de muertes maternas, el 10\% (43/431 muertes) correspondió a menores de 20 años, el 54,5\% (235/431 muertes) a mujeres entre 20-34 años y el $35,5 \%$ (153/431 muertes) a mujeres de 35 o más años. No se registraron muertes maternas en mujeres de 50 o más años y sólo 1 muerte en menores de 15 años.

La RMM de las 7 primeras causas de muerte según rangos de edad, mostró que la edad materna de 40 o más años fue un correlativo importante en 


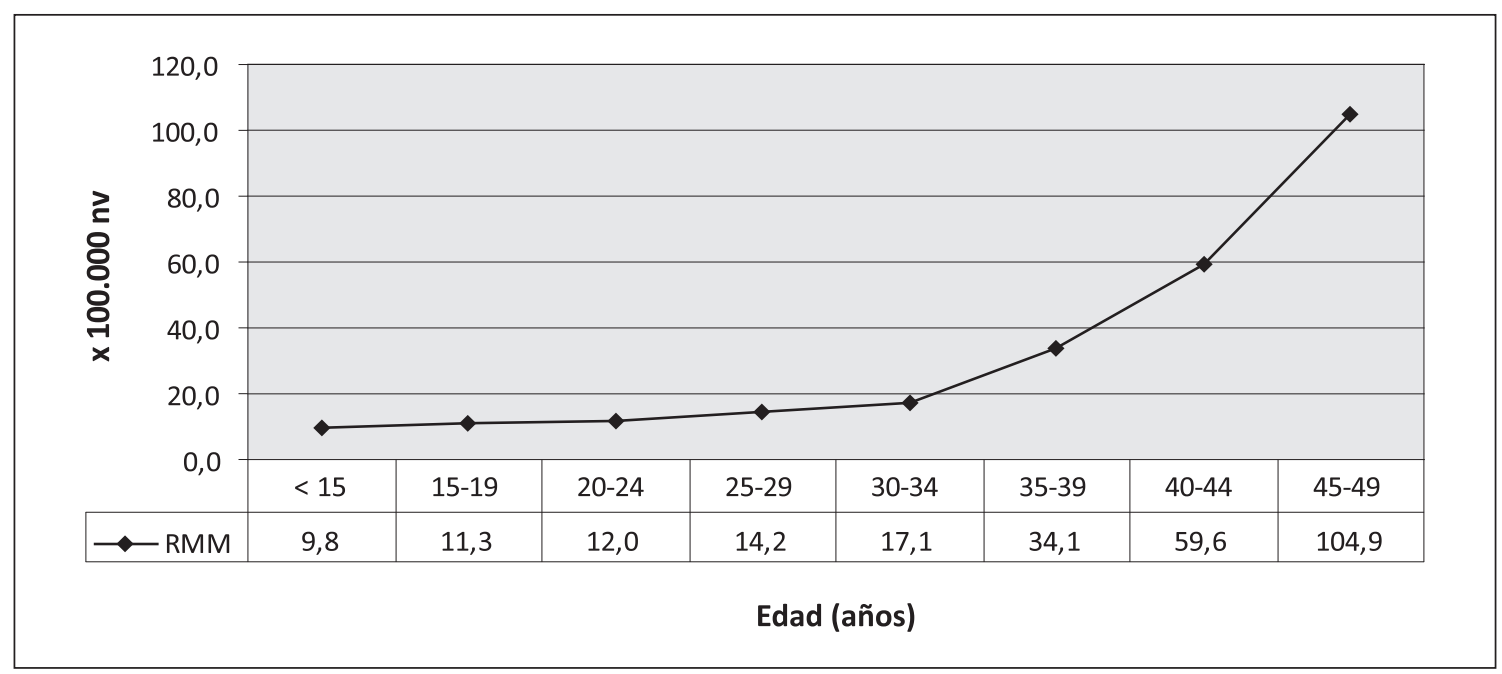

Figura 1. Tendencia de la mortalidad materna según rangos de edad, Chile 2000-2009.

Tabla 4. Razón de mortalidad materna (x 100.000 nv) según rangos de edad de las principales causas de muerte materna, Chile 2000-2009

\begin{tabular}{|cccccccc|}
\hline $\begin{array}{c}\text { Edad } \\
\text { (años) }\end{array}$ & $\begin{array}{c}\text { Enfermedades } \\
\text { concurrentes } \\
\text { (n: 115) }\end{array}$ & $\begin{array}{c}\text { Hipertensión } \\
\text { arterial } \\
(\mathbf{n}: \mathbf{1 0 5})\end{array}$ & $\begin{array}{c}\text { Aborto } \\
(\mathbf{n}: \mathbf{3 8})\end{array}$ & $\begin{array}{c}\text { Embolia } \\
\text { obstétrica } \\
\text { (n: 21) }\end{array}$ & $\begin{array}{c}\text { Hemorragia } \\
\text { posparto } \\
\text { (n: 20) }\end{array}$ & $\begin{array}{c}\text { Embarazo } \\
\text { ectópico } \\
\text { (n: 20) }\end{array}$ & $\begin{array}{c}\text { Sepsis } \\
\text { puerperal } \\
\text { (n: 16) }\end{array}$ \\
\hline$<15$ & --- & 9,83 & --- & --- & --- & --- & -- \\
\hline $15-19$ & 2,69 & 4,03 & 1,61 & 0,54 & 0,27 & --- & 0,27 \\
\hline $20-24$ & 4,65 & 3,04 & 0,36 & 0,54 & 0,36 & 0,36 & 0,36 \\
\hline $25-29$ & 2,78 & 4,17 & 2,43 & 0,52 & 0,69 & 0,69 & 0,87 \\
\hline $30-34$ & 4,19 & 3,79 & 0,80 & 0,80 & 1,00 & 1,40 & 0,80 \\
\hline $35-39$ & 7,08 & 7,76 & 3,37 & 2,70 & 1,35 & 2,36 & 1,01 \\
\hline $\mathbf{2}$ & 24,90 & 7,11 & 2,37 & 1,19 & 4,74 & --- & 1,19 \\
\hline
\end{tabular}

la mayoría de las causas de muerte, principalmente en la asociación con enfermedades concurrentes que alcanzó una RMM de 24,9/100.000 nv (Tabla 4).

\section{Discusión}

Durante el período 2000-2009, Chile no logró reducir la tendencia de la mortalidad materna, lo que dificulta el cumplimiento del $5^{\circ}$ objetivo del Milenio. Esto se debe al cambio del perfil epidemiológico y demográfico de la población obstétrica chilena, caracterizado por una natalidad general sin cambios, por el aumento significativo de los nacimientos en mujeres de 40 o más años y del aumento de las muertes maternas por enfermedades concurrentes.

El control poblacional de la fecundidad y el espaciamiento de los embarazos, son una de las principales estrategias para lograr el descenso de la morbilidad ${ }^{13,14}$ y mortalidad materna de un país ${ }^{15,16}$, porque permiten una menor exposición al riesgo de enfermar o morir, por causas inherentes al proceso reproductivo, como también por reducir las muertes por aborto inseguro ${ }^{17}$. Por el contrario, en Chile, en el período 2000-2009 el 31,8\% de los nacimientos proviene de población obstétrica 
de alto riesgo materno y perinatal (adolescentes y mujeres $>35$ años), que en conjunto contribuyeron con el 45,5\% (196/431) de las muertes maternas del período, sugiriendo una mala estrategia nacional de control de la fecundidad.

Previamente comunicamos el envejecimiento de la mujer chilena en edad fértil (período 19902004), con un aumento de $65,4 \%$ de la población de mujeres de 40-44 años y de su fecundidad en $51,7 \%{ }^{18}$. Adicionalmente demostramos el alto riesgo materno y perinatal en mujeres de 40 o más años, con mortalidad materna de 142,8/100.000 nv y con un riesgo 7,13 veces mayor de muerte materna comparadas con mujeres de 20-34 años ${ }^{19}$. También en España, se informó del aumento de la mortalidad materna asociada a la edad materna avanzada y a la postergación del embarazo, con aumento de la mortalidad materna de $20 \%$ en 2005 respecto a $1996^{20}$. Informes poblacionales de Europa y Estados Unidos de Norteamérica, confirman que el aumento de la edad materna es un factor emergente de riesgo demográfico para mortalidad materna ${ }^{21-25}$.

En esta investigación reportamos que en Chile, en el período 2000-2009, el 51,1\% de las muertes maternas se concentraron en las enfermedades maternas concurrentes y en las asociadas a hipertensión arterial, por lo que las estrategias destinadas a la reducción de la mortalidad materna para cumplir con el $5^{\circ}$ objetivo del Milenio deben estar destinadas especialmente al control de estas dos causas.

Previamente reportamos que en el período 1990-2000, la tendencia de la RMM por enfermedades concurrentes no presentó cambios significativos, siendo la principal causa de muerte materna en mujeres de 40 o más años, con una RMM de $15,3 / 100.000 \mathrm{nv}^{8}$, mientras que en el período 2000-2009, la RMM en estas mujeres aumentó a 24,9/100.000 nv. Las enfermedades concurrentes fueron la primera causa de muerte materna en el período 2000-2009, lo que puede explicarse por el aumento significativo de la natalidad en mujeres de 40 o más años y al envejecimiento de la mujer chilena en edad fértil. Sugerimos como estrategias para la reducción de las muertes maternas por enfermedades concurrentes, el control de la fecundidad y el consejo reproductivo pregestacional en estas mujeres.

La hipertensión arterial fue la segunda causa de muerte materna en el período 2000-2009; 46,7\% de ellas debidas a eclampsia, revelando un inadecuado control prenatal y manejo intrahospitalario, especialmente cuando la cobertura nacional del control prenatal y la atención profesional del parto superan el 90 y $99 \%$ respectivamente ${ }^{10}$. Las estrategias para la reducción de las muertes maternas asociadas a hipertensión arterial son el control de la fecundidad en mujeres en edades reproductivas extremas, como también la permanente capacitación de los profesionales para el correcto diagnóstico y tratamiento de esta patología.

El aborto fue la tercera causa de muerte materna en el período 2000-2009; siendo la única de las principales causas que presentó una tendencia descendente significativa en el período estudiado. En el período anterior $1990-2000^{8}$, también observamos una reducción en el número de muertes por aborto, probablemente en relación con el descenso significativo de la natalidad, lo que no sucedió en el período 2000-2009. La estrategia para continuar el descenso de la mortalidad materna por aborto es el control de la fecundidad, especialmente en grupos de mujeres de alto riesgo de recurrir al aborto inseguro ${ }^{26}$. Es posible que concurra a explicar la baja mortalidad materna por aborto, el uso de métodos abortivos de menor morbilidad y mortalidad, como es el misoprostol, del cual desconocemos su prevalencia de utilización. Según cifras del Ministerio de Salud en el año 2008, hubo 248.366 nacidos vivos y 33.428 egresos por aborto, de los cuales $11.070(33,1 \%)$ fueron calificados como "no especificados" (código O06) que muy posiblemente correspondan a abortos inducidos ${ }^{27}$, cifra muy inferior a los 160.000 abortos inducidos anuales estimados para Chile ${ }^{28}$. Es necesario destacar un estudio reciente que en base a un modelo epidemiológico, válido y reproducible, estimó que en Chile en 2008, sólo el 10,6\% de los egresos por aborto, corresponderían a complicaciones por aborto inducido ${ }^{29}$. Es importante resaltar que en la actualidad el riesgo absoluto de morir por aborto en Chile es de 0,046/100.000 mujeres de 15-49 años ( 1 en 2.000.000 mujeres de 15-49 años) ${ }^{30}$, como también que la legislación promulgada en 1989 y que prohíbe toda causal de aborto no ha influido en la tendencia de la mortalidad materna por aborto inseguro como ha sido sugerido ${ }^{31}$.

La embolia obstétrica fue la cuarta causa de muerte materna en el período 2000-2009, con una $\operatorname{RMM}(0,9 / 100.000 \mathrm{nv})$ similar a lo reportado en Estados Unidos de Norteamérica (1,0/100.000 
nv) $)^{32}$, Canadá $(0,5 / 100.000 \text { nv })^{33}$ y Reino Unido ${ }^{34}$. El aumento de la embolia obstétrica en Chile puede deberse a un mejor diagnóstico de la complicación, como también al envejecimiento poblacional de la mujer chilena en edad fértil ${ }^{18}$, dada su asociación con la edad materna avanza$\mathrm{da}^{35-38}$. La principal estrategia para la prevención del trombo-embolismo es el uso de heparina en mujeres con factores de riesgo ${ }^{37}$, sopesando el riesgo de trombosis versus el riesgo de complicaciones hemorrágicas por heparina, que puede alcanzar al $2 \%$ de las pacientes tratadas ${ }^{39}$.

La hemorragia del posparto fue la quinta causa de muerte materna en el período 2000-2009. En décadas anteriores a 1990 esta importante causa de muerte se vinculaba a la gran multiparidad y a la atención del parto por personal sin capacitación para resolver esta complicación. Actualmente, la gran multiparidad es inferior al $4 \%{ }^{11} \mathrm{y}$ los partos son asistidos institucionalmente casi en su totalidad por profesionales ${ }^{10}$. Es posible que la alta incidencia de operación cesárea en el país (rango: $27-83 \%)^{40}$, haya impedido la reducción de la mortalidad materna por hemorragia del posparto. La hemorragia severa del posparto tiene para parto vaginal una prevalencia de 2,94\% (IC 95\% 2,82$3,07$ ) y para cesárea de $6,38 \%$ (IC $95 \% 5,45-7,31)^{41}$, por lo que es importante establecer políticas conducentes a reducir la operación cesárea.

La muerte materna por embarazo ectópico comparte el quinto lugar con la hemorragia del posparto. La RMM por esta causa $(0,8 / 100.000 \mathrm{nv})$ duplica a la de Estados Unidos de Norteamérica $(0,4 / 100.000 \mathrm{nv})^{32}$. En 2008 hubo 3.543 egresos por embarazo ectópico ${ }^{27}$ y 3 muertes maternas $(0,08 \%)$, con una prevalencia $\sim 12,6 / 1.000$ embarazos, similar a Canadá que en el período 2004-2005 fue de 11,9/1.000 embarazos, con frecuencia que aumenta a partir de los 35 años $^{42}$. Lo anterior es consistente con lo ocurrido en Chile, en que la RMM más alta por embarazo ectópico correspondió al rango de edad materna entre 35-39 años $(2,4 / 100.000 \mathrm{nv})$.

La muerte materna por sepsis puerperal fue la sexta causa $(0,7 / 100.000 \mathrm{nv})$, siendo menor que la RMM que reportamos en el período 1990$2000(1,7 / 100.000 \mathrm{nv})^{8}$. La menor RMM del actual período es posible que sea el resultado de la atención intrahospitalaria y profesional de 99\% de los partos ${ }^{10}$, y del uso amplio de antibióticos profilácticos en situaciones de alto riesgo de in- fección puerperal, como son la operación cesárea y la rotura prematura de las membranas ovulares.

En Chile, a diferencia de otros países, la mortalidad materna en adolescentes menores de 15 años no presenta diferencias significativas comparadas con adultas de 20-34 años (OR = 1,56; IC95\%: 0,50 a 4,$31 ; \mathrm{p}=0,372)$, mientras que en adolescentes de 15-19 años la mortalidad materna es significativamente menor $(\mathrm{OR}=0,72$; IC95\%: 0,56 a 0,92; $\mathrm{p}<0,008)^{43}$. Pese a lo anterior, debemos reflexionar si debemos aceptar la muerte de niñas madres y las estrategias destinadas al retraso del inicio de la vida sexual. En el período 2000-2009, el 15,9\% de los nacimientos correspondieron a niñas menores de 20 años, con mortalidad materna de 11,3/100.000 nacidos vivos (43 muertes), inferior al promedio nacional, pero que corresponde al $10 \%$ del total de muertes maternas del período. Es evidente que hay que fortalecer las estrategias establecidas en las políticas públicas sanitarias y educacionales vigentes, destinadas a retrasar el inicio de la vida sexual, como también las destinadas a prevenir el embarazo no deseado, que en las adolescentes significa además, el abandono de su educación y la perpetuación de la pobreza. Por las razones anteriores debemos considerar como evitable la muerte materna producto del embarazo adolescente.

Chile es el país latinoamericano con menor mortalidad materna ${ }^{44}$, como consecuencia de su desarrollo social, económico y sanitario ${ }^{30}$. Los factores causales de muerte materna en Chile en la década 2000-2009, son diferentes a los presentados para América Latina y el Caribe por el grupo de trabajo integrado por representantes de WHO, UNFPA, World Bank y otros (hipertensión arterial $25,7 \%$, hemorragia $20,8 \%$, parto obstruido $13,4 \%$, aborto $12 \%$ y sepsis puerperal $7,7 \%$ ), y con mayor similitud a la de países desarrollados (hipertensión arterial $16,1 \%$, embolia $14,9 \%$, causas indirectas $14,4 \%$, hemorragia $13,4 \%$ y aborto $8,2 \%)^{45}$.

Chile comparte el compromiso mundial de reducir la RMM en 75\% (9,9/100.000 nv) para el período 1990-2015 (Quinto Objetivo del Milenio), lamentablemente nuestro análisis muestra que estamos lejos de la meta. Recientemente el Gobierno de Chile ha dado a conocer los objetivos sanitarios de la década 2011-2020 ${ }^{46}$, invitando a los equipos de salud a su logro; desafortunadamente, la normativa del Ministerio de Salud no indica ninguna estrategia orientada a reducir la mortalidad materna. De acuerdo a nuestros resultados proponemos 
Mortalidad materna en Chile no cambia entre los años 2000-2009 - E. Donoso et al

algunas sugerencias que debieran estar contenidas en un programa Ministerial para la Reducción de la Mortalidad Materna:

- Reducción de la natalidad en adolescentes, con programas efectivos de educación en sexualidad y prevención del embarazo.

- Adecuado consejo pregestacional a mujeres de 40 o más años.

- Adecuado consejo pregestacional a mujeres con enfermedades crónicas.

- Capacitación en planificación familiar a médicos internistas y de subespecialidades.

- Educación continua de matronas, enfermeras matronas y médicos generales, en identificación del alto riesgo obstétrico y su derivación oportuna al nivel secundario o terciario.

- Actualización cada 5 años de las Guías Clínicas Ministeriales Materno-Perinatal.

- Comité Ministerial de auditoría mensual de las muertes maternas.

- Incorporar en el certificado de defunción la sección Muerte Materna, etnia y nacionalidad de los fallecidos.

- Notificación obligatoria de los casos de near miss $^{47}$.

- Desmunicipalizar los programas de salud reproductiva.

\section{Conclusiones}

El aumento de la natalidad en mujeres en edad materna avanzada, grupo con una creciente tasa de mortalidad por enfermedades concurrentes, dificultará el cumplimiento del $5^{\circ}$ objetivo del Milenio. Las estrategias para lograr ese objetivo deben centrarse en el control de la fecundidad de las mujeres con enfermedades crónicas y en el manejo del síndrome hipertensivo del embarazo, responsables de $51,1 \%$ de las muertes maternas en Chile, ambas estrechamente asociadas al embarazo en edad materna avanzada.

\section{Referencias}

1. Countdown Coverage Writing Group; Countdown to 2015 Core Group, Bryce J, Daelmans B, Dwivedi A, Fa uveau V, Lawn JE, Mason E, Newby H, Sh ankar A, Starrs A, Wardlaw T. Co untdown to 2015 for maternal, newborn, and child survival: the 2008 report on tracking coverage of interventions. Mancet 2008; 371: 1247-58.
2. Hill K, Thomas K, AbouZahar C, Walter N, Say L, Inoue M, Suzuki E, on behalf of the Maternal Mortality Working Group. Estimates of maternal mortality worldwide between 1990 and 2005: an assessment of available data. Lancet 2007; 370: 1311-9.

3. AbouZahr C. Safe motherhood: a brief history of the global movement 1947-2002. Br Med Bull 2003; 67: 1325.

4. United Nations. United Nations Millennium Declaration. Resolution adapted by the General Assembly, 55th Session of the United Nations General Assembly, New York, September 18, 2000.

5. Viel B, Campos W. Chilean history of infant and maternal mortality, 1940-1985. Perspective Int Planif Fam 1987; Spec No: 24-8.

6. Armijo R, Monreal T. [The problem of induced abortion in Chile. Bol Oficina Sanit Panam 1966; 60 (1): 39-45. [Article in Spanish].

7. Szot J, Moreno C. Mortalidad por aborto en Chile: análisis epidemiológico 1985-2000. Rev Chil Obstet Ginecol 2003; 68: 309-14.

8. Donoso E. [The reduction in maternal mortality in Chile, 1990-2000]. Rev Panam Salud Publica/Pan Am J Public Health 2004; 15 (5): 326-30. [Article in Spanish].

9. OPS/OMS. Plan de acción regional para la reducción de la mortalidad materna en las Américas. Bol Oficina Sanit Panam 1991: 110: 448-54.

10. Gobierno de Chile. Ministerio de Salud. Departamento de Epidemiología. Objetivos Sanitarios para la década 2000-2010. Primera Edición. Octubre, 2002. Disponible en: http://www.redsalud.gov.cl/portal/url/item/6bdb73 323d19be93e04001011f013325.pdf.][Consultado el 5 de abril de 2011].

11. Instituto Nacional de Estadísticas (INE). Anuarios de Estadísticas Vitales de Chile, 2000-2009.

12. Organización Mundial de la Salud (OMS), Organización Panamericana de la Salud (OPS). Clasificación Estadística Internacional de Enfermedades y Problemas Relacionados con la Salud. Décima Revisión. Volumen 1. Publicación Científica No 554. Washington DC, 1995. Disponible en: http://apps.who.int/classifications/apps/ icd/icd10online/. [Consultado el 3 de febrero de 2011].

13. Conde-Agudelo A, Ro as-Bermúdez A, Ka fury-Goeta AC. Effects of birth spacing on maternal health: a systematic review. Am J Obstet Gynecol 2007; 196 (4): 297-308

14. Yeakey MP, Muntifering CJ, Ramachandran DV, My int Y, Creanga AA, Ts ui AO. How contraceptive use affects birth intervals: results of a literature review. Stud Fam Plann 2009; 40 (3): 205-14.

15. Conde-Agudelo A,Be izán JM.Maternal morbidity and 
mortality associated with interpregnancy interval: cross sectional study. BM 2000; 321 (7271): 1255-9.

16. Stover J, Ross J. How increased contraceptive use has reduced maternal mortality. Matern Child Health J 2010; 14 (5): 687-95.

17. WHO. Unsafe Abortion. Global and regional estimates of the incidence of unsafe abortion and associated mortality in 2003. Fifth edition. World Health Organization, 2007. Geneva, Switzerland. Disponible en: http://whqlibdoc.who.int/publications/2007/9789241596121_eng.pdf. [Consultado el 5 de abril de 2011].

18. Donoso E, Carvajal J, Domínguez MA. [Hecundity reduction and aging in fertile women population in the period 1990-2004 in Chile] Rev Med Chile 2009; 137: 766-73. [Article in Spanish].

19. Donoso E, Villarroel L. [Reproductive risk of women over 40 years old]. Rev Med Chile 2003; 131: 55-9. [Article in Spanish].

20. Fernández MA, Cavanillas AB, Dramaix-Wilmet M, Soria FS, de Mata Donado Campos J, Guibert DH. Increase in maternal mortality associated with change in the reproductive pattern in Spain: 1996-2005. J Epidemiol Community Health 2009; 63 (6): 433-8.

21. Sa lanave B, Bo uvier-Colle MH. The likely increase in maternal mortality rates in the United Kingdom and in France until 2005. Paediatr Perinat Epidemiol 1 996; 10 (4): 418-22.

22. Alexander S, Wildman K, Zhang W, Langer M, Vutuc C, Lindmark G. Maternal health outcome in Europe. Eur J Obstet Gynecol Reprod Biol 2003; 111: S78-S87.

23. Temmerman M, Verstraelen H, Martens G, Bekaert A. Delayed chilbearing and maternal mortality. Eur J Obstet Gynecol Reprod Biol 2004; 114 (1): 19-22.

24. Lang CT, King JC. Maternal mortality in the United States. Best Pract Res Clin Obstet Gynaecol 2008; 22 (3): 517-31.

25. Schutte JM, Steegers EAP, Schuitemaker NWE, Santema JG, de Boer K, Vermeulen G, et al, the Netherlands Maternal Committe. Rise in maternal mortality in the Netherlands. BJOG 2010; 117: 399-406.

26. Langer A. [U nwanted pregnancy: impact on health and society in Latin America and the Caribbean]. Rev Panam Salud Publica 2002; 11 (3): 192-204. [Article in Spanish].

27. Gobierno de Chile, Ministerio de Salud. Orientaciones Técnicas. Para la atención integral de mujeres que presentan un aborto y otras pérdidas reproductivas. Disponible en: http://www.minsal.gob.cl/portal/url/item/ b53faf5d1d527a52e04001011e010ef5.pdf. [Consultado el 15 de mayo de 2012].

28. Singh S, Wulf D. An overview of clandestine abortion in Latin America. Issues in brief. The Alan Guttmacher Ins- titute. 1996. Disponible en: http://www.guttmacher.org/ pubs/ib12sp.pdf. [Consultado el 15 de mayo de 2012].

29. Koch E, Bravo M, Gatica S, Stecher JF, Aracena P, Valenzuela S, Ahlers I. Sobrestimación del aborto inducido en Colombia y otros países latinoamericanos. Ginecol Obstet Mex 2012; 80 (5): 360-72.

30. Koch E, Thorp J, Bravo M, Gatica S, Romero CX, Aguilera $\mathrm{H}$, et al. Women's education level, maternal health facilities, abortion legislation and maternal deaths: a natural experiment in Chile from 1957 to 2007. PLoS One 2012; 7 (5): e36613.

31. Shepard BL, Casas Becerra L. Abortion policies and practices in Chile: ambiguities and dilemmas. Reprod Health Matters 2007; 15 (30): 202-10.

32. Heron M, Hoyert DL, Murphy SL, Xu J, Kochanek KD, Tejada-Vera B. Deaths: final data for 2006. Natl Vital Stat Rep 2009; 57 (14): 1-134.

33. Bartholomew S, Liston R, O`Brien B. Maternal health outcome. 15. Maternal Mortality Ratio. En: Public Health Agency of Canada. Canadian Perinatal Health Report, 2008 Edition. Ottawa 2008. Disponible en: http://www.publichealth.gc.ca/cphr] [Consultado el 5 de abril de 2011].

34. Neilson JP. Maternal mortality. Current Obstet Gynaecol 2005; 15: 375-81.

35. Drife J. Thrombosis and thromboembolism. Chapter 2. Why mothers die, 2000-2002 report. Confidencial inquieries into maternal deaths [on line]; 2004. Disponible en: http://www.cmace.org.uk/getdoc/b36df07d-8c9e40cf-95bd-dc5173c957f7/Chapter2.aspx. [Consultado el 3 de febrero de 2011].

36. Abenhaim HA, Azoulay L, Kramer MS, Leduc L. Incidence and risk factors of amniotic fluid embolisms: a population-based study on 3 million births in the United States. Am J Obstet Gynecol 2008; 199 (1): 49.e1-8.

37. James AH. Venous thromboembolism in pregnancy. Arterioscler Thromb Vasc Biol 2009; 29 (3): 326-31.

38. Conde-Agudelo A, Romero R.Amniotic fluid embolism: an evidence-based review. Am J Obstet Gynecol 2009; 201(5): 445.e1-13.

39. Greer IA, Ne son-Piercy C. Low-molecular-weight heparins for thromboprophylaxis and treatment of venous thromboembolism in pregnancy: a systematic review of safety and efficacy. Blood 2005; 106 (2): 401-7.

40. Murray SF. Relation between private health insurance and high rates of caesarean section in Chile: qualitative and quantitative study. BM 2000; 321 (7275): 1501-5.

41. Carroli G, Cuesta C, Abalos E, Gulmezoglu AM. Ep demiology of postpartum haemorrhage: a systematic review. Best Pract Res Clin Obstet Gynaecol 2008; 22 (6): 999-1012. 
42. McCourt C, Wei S, Fraser W. Maternal health outcome. 18. Rate of ectopic pregnancy. En: Public Health Agency of Canada. Canadian Perinatal Health Report, 2008 Edition. Ottawa 2008. Disponible en: http://www.publichealth.gc.ca/cphr/. Consultado el 5 de febrero de 2011.

43. Donoso E, Becker J, Villarroel L. [Birth rates and reproductive risk in adolescents in Chile, 1990-1999]. Bev Panam Salud Pública 2003; 14 (1): 3-8. [Article in Spanish].

44. OPS. Situación de salud en las Américas. Indicadores Básicos 2009. Disponible en: http://new.paho.org/hq dmdocuments/2009/IB_SPA_2009.pdf. [Consultado el
2 de noviembre de 2011].

45. Khan KS, Wojdyla D, Say L, Gülmezoglu AM, Van Look PF. WHO analysis of causes of maternal death: a systematic review. Mancet 2006; 367 (9516): 1066-74.

46. MINSAL. Estrategia nacional de salud para el cumplimiento de los objetivos sanitarios de la década 20112020. Disponible en: http://www.minsal.gob.cl/portal/ docs/1/5648346.pdf.] [Consultado el 16 de diciembre de 2011].

47. Tunçalp Ö, Hindin MJ, Souza JP, Chou D, Say L. The prevalence of maternal near miss: a systematic review. BJOG 2012; 119: 653-661. 